\title{
Association of Epicardial Adipose Tissue Thickness by Echocardiography With Coronary Artery Disease
}

\author{
Surendra Naik ${ }^{1}$, Nitish Naik $^{1}$, Niraj Pandey ${ }^{1}$, Ashish Upadhyay ${ }^{1}$, and Ambuj Roy ${ }^{1}$ \\ ${ }^{1}$ All India Institute of Medical Sciences
}

June 17, 2021

\begin{abstract}
Background: Epicardial adipose tissue (EAT) mimics visceral fat which is associated with metabolic derangements and coronary artery disease (CAD). EAT volume (EAT-V) measured by CT scan had shown good correlation with CAD. QRISK3 score is a validated risk predictor of future cardiovascular events but has limitations. We assessed whether EAT thickness (EAT-T) measured by echocardiography, a simple and widely available tool, correlated with EAT-V, and whether EAT-T is a predictor of CAD independently of QRISK3 scores. Methods: We enrolled 97 patients who underwent CTA for evaluation of chest pain. EAT-T was measured by 2D-echocardiography in parasternal long axis (PLAX) and parasternal short axis (PSAX) views. We evaluated association of EAT-T with EAT-V and CAD ([?]50\% stenosis on CTA); and independent predictive value of EAT-T for CAD after adjusting for QRISK 3 scores. Results: EAT-T was significantly more in patients with CAD (PLAX: $4.82 \pm$ $1.31 \mathrm{~mm}$ vs. $4.06 \pm 1.25 \mathrm{~mm}, \mathrm{p}=0.005)$. EAT-T correlated strongly with EAT-V $(\mathrm{r}=0.75, \mathrm{p}<0.001)$. On receiver operating characteristic curve analysis, EAT-T (PLAX) [?]3.9 mm (area-under-curve: 0.68; 95\% CI: 0.58-0.79, sensitivity 84\%, specificity $55 \%)$ predicted the presence of CAD. On multivariate analysis after adjusting for QRISK 3 scores, EAT-T showed significant association with CAD with highest odds ratio for indexed EAT-T (EAT-T/body surface area) (PLAX) [?]2.2 mm/m2 (OR 5.40; 95\% CI: 2.17-13.55.; $\mathrm{p}<0.001)$. Conclusion: EAT-T is a predictor of CAD independent of QRISK3 scores. An increased EAT-T detected CAD with $>80 \%$ sensitivity. These findings need to be validated in larger prospective cohort studies.
\end{abstract}

Title: Association of Epicardial Adipose Tissue Thickness by Echocardiography With Coronary Artery Disease

Running title: Epicardial Adipose Tissue in Coronary Artery Disease

\section{Authors:}

1. Surendra Kumar Naik ${ }^{1}, \mathrm{MD}, \mathrm{DM}$

2. Nitish Naik ${ }^{1}, \mathrm{MD}, \mathrm{DM}$

3. Niraj Nirmal Pandey ${ }^{2}$, MD, DM

4. Ashish Datt Upadhyay ${ }^{3}, \mathrm{PhD}$

5. Ambuj Roy ${ }^{1}, \mathrm{MD}, \mathrm{DM}$

\section{Affiliations:}

${ }^{1}$ Department of Cardiology, All India Institute of Medical Sciences, New Delhi, India

${ }^{2}$ Department of Cardiovascular Radiology and Endovascular Interventions, All India Institute of Medical Sciences, New Delhi, India

${ }^{3}$ Department of Biostatistics, All India Institute of Medical Sciences, New Delhi, India

\section{Funding:}


Not applicable

\section{Competing interests}

The authors have no competing interests to declare.

\section{Corresponding author:}

Dr Ambuj Roy, MD, DM, FACC, FRCP

Professor,

Department of Cardiology,

Cardio Thoracic Sciences Centre, All India Institute of Medical Sciences,

New Delhi (110029), India.

Ph no.: +91-9810992822

E-mail: drambujroy@gmail.com

Abstract:

Background: Epicardial adipose tissue (EAT) mimics visceral fat which is associated with metabolic derangements and coronary artery disease (CAD). EAT volume (EAT-V) measured by CT scan had shown good correlation with CAD. QRISK3 score is a validated risk predictor of future cardiovascular events but has limitations. We assessed whether EAT thickness (EAT-T) measured by echocardiography, a simple and widely available tool, correlated with EAT-V, and whether EAT-T is a predictor of CAD independently of QRISK3 scores.

Methods: We enrolled 97 patients who underwent CTA for evaluation of chest pain. EAT-T was measured by 2D-echocardiography in parasternal long axis (PLAX) and parasternal short axis (PSAX) views. We evaluated association of EAT-T with EAT-V and CAD ([?]50\% stenosis on CTA); and independent predictive value of EAT-T for CAD after adjusting for QRISK 3 scores.

Results: EAT-T was significantly more in patients with CAD (PLAX: $4.82 \pm 1.31 \mathrm{~mm}$ vs. $4.06 \pm 1.25 \mathrm{~mm}$, $\mathrm{p}=0.005)$. EAT-T correlated strongly with EAT-V $(\mathrm{r}=0.75, \mathrm{p}<0.001)$. On receiver operating characteristic curve analysis, EAT-T (PLAX) [?]3.9 mm (area-under-curve: 0.68; 95\% CI: 0.58-0.79, sensitivity 84\%, specificity 55\%) predicted the presence of CAD. On multivariate analysis after adjusting for QRISK 3 scores, EAT-T showed significant association with CAD with highest odds ratio for indexed EAT-T (EAT-T/body surface area) (PLAX) [?]2.2 mm/m² (OR 5.40; 95\% CI: 2.17-13.55.; $\mathrm{p}<0.001)$.

Conclusion: EAT-T is a predictor of CAD independent of QRISK3 scores. An increased EAT-T detected CAD with $>80 \%$ sensitivity. These findings need to be validated in larger prospective cohort studies.

Keywords:

Epicardial Adipose Tissue Thickness; Epicardial Adipose Tissue Volume; Echocardiography; QRISK3 Score

\section{Introduction:}

Coronary artery disease (CAD) occurs $5-10$ years earlier in Indians ${ }^{(1)}$. Risk stratification using risk calculators like QRISK3 score is a key element in the efforts to prevent premature CAD, and to improve targeted implementation of preventive therapies for CAD. Limitations and lack of validation of these risk scores in local population lead to need for simple and widely accessible novel risk markers to provide a clinically meaningful improvement as a screening tool across the population.

Visceral adipose tissue deposition is a documented risk factor for cardiovascular diseases especially among Indians ${ }^{(2)}$. Epicardial adipose tissue (EAT) mimics visceral fat which is present between epicardium and visceral pericardium, and is associated with metabolic derangements and increased cardiovascular risk. EAT 
is implicated as an independent risk for CAD because of its proximity to the adventitia of major epicardial coronary arteries and has adverse autocrine and paracrine effect on coronary arteries ${ }^{(3,4)}$. EAT, including epicardial adipose tissue thickness (EAT-T) by echocardiography and epicardial adipose tissue volume (EAT$\mathrm{V})$ by computed tomography angiography (CTA) have been associated with $\mathrm{CAD}^{(5)}$. Thus, it may have incremental benefits in assessment of CAD risk and needs to be studied in local population.

Epicardial adipose tissue can be measured by three imaging modalities [CTA, magnetic resonance imaging (MRI) and echocardiography $]^{(6)}$. Each of these modalities has their attending benefits as well as limitations. CTA has been increasingly used recently because of its high spatial resolution which results in precise measurements of EAT-T as well as three-dimensional EAT volume (EAT-V), along with concurrent assessments of coronary artery calcium (CAC) score, coronary artery stenosis and high-risk plaque features (HRPF). However, CT is limited by the associated ionizing radiation related risks, relatively high cost, and limited availability. MRI avoids radiation exposure. However, limited availability and high costs limit the widespread use of MRI. Echocardiography is the widest available modality and a relatively cheaper method to measure EAT-T. Echocardiography also provides information on cardiac functions, has no radiation exposure, and easy to perform.

Hence, we tried to evaluate the correlation between echocardiographic EAT-T [and indexed EAT-T (EAT$\mathrm{T} /$ body surface area)] and EAT-V by CTA to know whether EAT-T could be used as surrogate marker for EAT-V, and whether EAT-T and indexed EAT-T are independent predictors of CAD in a multivariate analysis with QRISK3 score.

\section{Methods:}

We enrolled 97 consecutive patients with at least one cardiovascular risk factor [Hypertension (blood pressure [?]130/80 mmHg or on anti-hypertensive treatment), Type 2 Diabetes mellitus (Hba1c [?]6.5 or on antidiabetic treatment), dyslipidaemia (total cholesterol [?]200 mg/dl or LDL-C [?]130 mg/dl or on lipid lowering drugs) or Tobacco use (current smoker or tobacco chewing)] who were undergoing CTA for chest pain evaluation over a period of 12 months (January 2019 to February 2020) at the Cardio Thoracic Centre, All India Institute of Medical Sciences (AIIMS) New Delhi. Transthoracic echocardiography was performed in all patients for EAT-T measurements in parasternal long axis (PLAX) and parasternal short axis (PSAX) view, while CTA was reviewed for CAD, CAC score and EAT-V measurements. All patients included were assessed with detailed history, clinical examination and biochemical tests (blood sugar, blood pressure, lipid profile). The exclusion criteria were: (a) patients with known CAD, (b) patients with un-interpretable CT quality, (c) patients with poor echo window, and (d) those not consenting to participate.

\subsection{Measurements of EAT-V, CAC score and CAD definition on CTA:}

EAT-V was measured on the non-contrast CT images using a semi-automated technique on a dedicated workstation (Syngo.via; Siemens Healthcare, Forchheim, Germany). The region of interest (ROI) was manually drawn in all cases by tracing the pericardium in axial sections at $1 \mathrm{~cm}$ intervals, from the level of origin of left main coronary artery cranially to the cardiac apex (just below the level of posterior descending artery) caudally. Attenuation thresholds of -30 and $-250 \mathrm{HU}$ were applied within the ROI to identify adipose tissue-containing voxels.

CAC score was calculated on the non-contrast CT images using the Agatston method (7) using a semiautomated technique (Application: Calcium scoring; syngo.via, Siemens Healthcare, Forchheim, Germany).

CAD was defined as presence of $>50 \%$ diameter stenosis in any of the coronary arteries on contrast-enhanced CTA.

\subsection{Measurements of EAT-T by transthoracic echocardiography:}

Transthoracic two-dimensional echocardiography was performed using standard technique in left lateral decubitus position on Philips EPIQ 7C system. EAT was defined as hypoechoic space between the linear hyperechoic visceral pericardium and epicardium. EAT-T was measured perpendicularly over the right 
ventricular free wall from both parasternal long-axis (PLAX) and parasternal short-axis (PSAX) view at end-systole in three cardiac cycles. Maximum value perpendicular to the aortic annulus for PLAX view (Figure 1 ) and perpendicular to the inter-ventricular septum at the mid-chordal level for PSAX view (Figure 2 ) was measured and average of three measurements was taken. Echocardiography was recorded and interpreted by observer who was blinded to CT findings, first 25 recordings was read by 2 observers for assessment of inter-observer variability (intra class correlation coefficient, 0.867; 95\% CI, 0.699-0.921; $\mathrm{P}<0.001)$.

\subsection{Statistical analysis:}

The quantitative variable/s was summarized through descriptive statistic mean and standard deviation (SD), and Inter-Quartile Range. The categorical variable/s was summarized through frequency (\%). Comparison of continuous and categorical variables was performed using unpaired t test and chi square test respectively. Strength of correlation between continuous variables like EAT-T by echocardiography and EAT-V by was assessed by Pearson's correlation coefficient. Univariate regression analysis and multivariate logistic regression analysis was done with EAT-T and indexed EAT-T as predictors to assess association with CAD. Cut-off value of EAT-T for diagnosis of CAD was assessed using receiver operating characteristic (ROC) curve analysis and area under the curve (AUC) by Youden index. $\mathrm{P}<0.05$ indicated statistical significance.

\section{Results:}

Baseline characteristics of the study participants are shown inTable 1 . Out of these 97 recruited patients, 38 had CAD based on the criteria defined in methods. CAD patients more often had significant CAC ([?]100 score) as compared to non-CAD patients (29\% vs $1.7 \%$, p value: 0.001 ). QRISK3 score was significantly more in $\mathrm{CAD}$ vs non-CAD patients (17.8\% vs $12.4 \%$, p value: 0.007$)$.

EAT-T measured by echocardiography, as well as EAT-V measured by CTA was significantly more in CAD patients as compared to non-CAD patients (Table 2 ). EAT-V measured by CTA showed significant correlation with EAT-T measured by echocardiography, both absolute and indexed, as shown in Table $\mathbf{3}$ and Table4 .

On receiver operating characteristic (ROC) curve analysis, EAT-T (PLAX) [?]3.9 mm (AUC 0.68; 95\% CI 0.58-0.79, sensitivity of $84 \%$ and specificity of $55 \%$ ), EAT-T (PSAX ) [?] $3.8 \mathrm{~mm}$ (AUC $0.66 ; 95 \%$ CI $0.56-$ 0.78 , sensitivity of $84 \%$ and specificity of $50 \%$ ), indexed EAT-T (PLAX) [?]2.2 mm/ $\mathrm{m}^{2}$ (AUC 0.69; $95 \%$ CI 0.59-0.80, sensitivity of $85 \%$ and specificity of $50 \%$ ), and indexed EAT-T (PSAX) [?]2.1 mm/ $\mathrm{m}^{2}$ (AUC $0.67 ; 95 \%$ CI $0.57-0.78$, sensitivity of $89 \%$ and specificity of $44 \%$ ) predicted the presence of CAD (Figure 3 ). Similarly, EAT-V [?]101 $\mathrm{cm}^{3}$ (AUC $0.73 ; 95 \%$ CI $0.64-0.84$, sensitivity of $92 \%$ and specificity of $51 \%$ ) and indexed EAT-V [?] $60 \mathrm{~cm}^{3} / \mathrm{m}^{2}$ (AUC $0.76 ; 95 \%$ CI $0.68-0.86$, sensitivity of $92 \%$ and specificity of $59 \%$ ) predicted the presence of CAD (Figure 3 ).

On univariate regression analysis EAT-T by PLAX (absolute value [?]3.9 $\mathrm{mm}$ and indexed value [?] 2.2 $\mathrm{mm} / \mathrm{m}^{2}$ ) and by PSAX (absolute value [?] $3.8 \mathrm{~mm}$ and indexed value [?]2.1 $\mathrm{mm} / \mathrm{m}^{2}$ ) showed significant association with CAD with odds ratios for CAD as shown inTable $\mathbf{5}$.

Multivariate regression analysis with QRISK3 score revealed that EAT-T by PLAX and PSAX (both absolute as well as indexed value) showed significant association with CAD (Table 6 ). The odds ratio of having CAD at EAT-T (PLAX) [?]3.9 $\mathrm{mm}$ and EAT-T (PSAX) [?] $3.8 \mathrm{~mm}$ was 2.8 (95\% CI 1.15-6.75, p=0.02) and 2.6 (95\% CI 1.07-6.03, $\mathrm{p}=0.03)$ respectively. Similarly, the odds ratio of indexed EAT-T (PLAX) [?] 2.2 $\mathrm{mm} / \mathrm{m}^{2}$ and indexed EAT-T (PSAX) $[?] 2.1 \mathrm{~mm} / \mathrm{m}^{2}$ was $5.4(95 \%$ CI $2.17-13.55, \mathrm{p}<0.001)$ and $3.3(95 \%$ CI $1.37-7.93, \mathrm{p}=0.01)$ respectively.

\section{Discussion:}

Patients with CAD had higher values of EAT. EAT-T by echocardiography correlated with EAT-V by CTA and was an independent predictor of CAD. Individuals with an EAT-T (PLAX) [?]3.9 mm and EAT$\mathrm{T}$ (PSAX) [?]3.8 mm had Odd's ratio of 2.8 and 2.6 respectively of having CAD. The sensitivity of these 
parameters to detect CAD was over $80 \%$ with specificity in the range of $50 \%$. The marker was an independent risk factor after adjusting for clinical risk score of QRISK 3 which includes traditional risk factors.

Previous studies have shown positive correlation of EAT with indices of obesity like BMI ${ }^{(8)}$. So, to nullify the effect of obesity on EAT and for better refinement of data, its indexed value was also evaluated in our study. Odds ratio of having CAD was higher for indexed EAT-T values as compared to absolute values in our study, which further signified the importance of neutralizing the effect of obesity on EAT measurement by indexing it to body surface area.

Epicardial adipose tissue surrounding the heart has been postulated in contributing to the development of coronary atherosclerosis by various mechanisms ${ }^{(3)}$. One such mechanism is EAT acting as inflammatory organ leading to local secretion of various adipo-cytokines and inflammatory mediators like tumour necrosis factor, interleukin- 6 and free fatty acids from epicardial adipocytes which may adversely affect coronary arteries ${ }^{(9,10)}$. Recent studies have also found that EAT was associated with the presence of high-risk vulnerable plaques ${ }^{(11)}$.

EAT-V measured by CTA in multiple studies has been associated with CAD. Iwasaki et al ${ }^{(12)}$ measured EAT-V in patients who underwent CTA and found that patients with significant coronary artery stenosis had higher EAT-V. K Yamashita et al ${ }^{(13)}$ showed that EAT-V was associated with total coronary plaque burden and vulnerable lipid rich necrotic plaques, and Ueno et $\mathrm{al}^{(14)}$ showed independent association of chronic total occlusions with EAT-V. A recent study by Wenji Yu et al in Chinese population also revealed association of EAT volume to obstructive $\mathrm{CAD}^{(15)}$. Thus EAT-V has emerged as a reliable risk predictor of CAD, plaque burden and vulnerable plaques, and EAT-T by echocardiography in our study showed good correlation with it.

On the other hand, echocardiographic EAT-T has not been widely studied due to the proposed limitations of measurement in linear dimensions, inter-observer variation and lack of good acoustic windows ${ }^{(16,17)}$. There are only few studies of EAT-T by echocardiography and have shown its association with CAD severity (assessed by various scores like Gensini score, SYNTAX score, and number of epicardial vessels involved), and with the presence of CAD (cut off being taken from $50 \%$ to $70 \%$ diameter stenosis) ${ }^{(18,19)}$. Verma B. et al showed that EAT-T has positive correlation with waist circumference, LDL-C level, Gensini score, and SYNTAX score, and also showed that EAT-T is independent predictor of CAD after adjusting for all conventional risk factors ${ }^{(20)}$. All these studies have adjusted for conventional risk factors in a multivariate model for showing EAT-T as independent predictor of CAD. However, in our study we included QRISK3 score in multivariate model. Since calculation of QRISK3 score require all conventional risk factors and is a better predictor of $\mathrm{CAD}$ and future $\mathrm{CV}$ events than individual conventional risk factor, therefore putting it in a multivariate model enhanced the independent predictive value of EAT-T.

Erkan et al observed mean EAT values of $4.3+-0.9 \mathrm{~mm}, 5.2+-1.5 \mathrm{~mm}$, and $7.5+-1.9 \mathrm{~mm}$ in patients with normal coronary arteries, minimal CAD, and significant CAD, respectively. In that study, a EAT cut-off value of $5.8 \mathrm{~mm}$ predicted significant CAD (AUC: 0.875; P < 0.001, 95\% CI: 0.825-0.926) in that study ${ }^{(21)}$. While various other studies found a threshold of 6-7 mm as elevated EAT-T ${ }^{(22,23)}$. In our study, optimum EAT cut-off value to predict CAD was identified as [?]3.9 mm on PLAX view (AUC: $0.68,95 \%$ CI: 0.58-0.79) with a sensitivity of $84 \%$ and a specificity of $55 \%$, respectively. These variations in EAT cut-offs in different studies may be explained by ethnic differences in the threshold for association with CAD as described by Moharram et al (24).

Our study had the following limitations. This was a single centre study with moderate number of patients. The above findings and cut-offs need to be validated in larger prospective cohort studies. The measurement of EAT is operator-dependent and inter-observer variations may occur; although we observed good interobserver correlation. Also measuring EAT-T in obese and those with sub-optimal echo windows is a challenge, these patients were excluded from this study. Also, this was a hospital-based study and may not reflect the normal population. However, a CT scan-based study to show correlation with CAD may not be feasible in normal population. 


\section{Conclusion:}

EAT thickness measured by transthoracic echocardiography correlated with CTA-based EAT volume. The EAT thickness was an independent predictor of CAD and a sensitive tool to diagnose CAD in Indian population. However, larger prospective studies will be needed to validate these findings.

\section{Data Availability Statement:}

The data that support the findings of this study are available from the corresponding author upon reasonable request.

\section{Ethics and consent:}

This study had been approved by Institute Ethics Committee (viz. Ref. No.: IECPG-52/28.02.2018, RT19/14.11.2018) and written informed consents were taken from all patients.

\section{Competing interests:}

The authors have no competing interests to declare.

\section{Author Contributions:}

SKN, NN, NNP, ADU and AR contributed to the concept, design, acquisition, analysis and interpretation of data. NN and AR drafted the work and revised it critically for important intellectual content. AR did the final approval of the version to be published. SKN was accountable for all aspects of the work ensuring that accuracy or integrity of any part of the work were appropriately investigated and resolved.

\section{References:}

1. Prabhakaran D, Jeemon P, Roy A. Cardiovascular Diseases in India, Current Epidemiology and Future Directions. Circulation. 2016; 133: 1605-1620.

2. Banerji MA, Faridi N, Atluri R, et al. Body Composition, Visceral Fat, Leptin, and Insulin Resistance in Asian Indian Men. The Journal of Clinical Endocrinology \& Metabolism. 1999; 84(1): 137-144.

3. Christensen RH, Johan Von Scholten B, Lehrskov LL, et al. Epicardial adipose tissue: an emerging biomarker of cardiovascular complications in type 2 diabetes? Ther Adv Endocrinol Metab. 2020; 11: 2042018820928824.

4. Mazurek T, Zhang L, Zalewski A, et al. Human EAT is source of inflammatory mediators. Circulation. 2003; 108: 2460-2466.

5. Kim BJ, Kim BS, Kang JH. Echocardiographic epicardial fat thickness is associated with coronary artery calcification - results from the CAESAR study. Circ J. 2015; 79: 818-824.

6. Bertaso AG, Bertol D, Duncan BB, et al. Epicardial Fat: Definition, Measurements and Systematic Review of Main Outcomes. Arq Bras Cardiol. 2013; 101(1): e18-e28.

7. Agatston AS, Janowitz WR, Hildner FJ, et al. Quantification of coronary artery calcium using ultrafast computed tomography. J Am Coll Cardiol. 1990; 15: 827-32.

8. Rabkin SW. The relationship between epicardial fat and indices of obesity and the metabolic syndrome: a systematic review and meta-analysis. Metab Syndr Relat Disord. 2014; 12(1): 31-42.

9. Verhagen SN, Visseren FL. Perivascular adipose tissue as a cause of atherosclerosis. Atherosclerosis. 2011; 214: 3-10.

10. Weyer C, Foley JE, Bogardus C, et al. Enlarged subcutaneous abdominal adipocyte size, but not obesity itself, predicts type II diabetes independent of insulin resistance. Diabetologia. 2000; 43: 1498 $-1506$.

11. Alexopoulos N, McLean DS, Janik M, et al. Epicardial adipose tissue and coronary artery plaque characteristics. Atherosclerosis. 2010; 210: $150-154$.

12. Iwasaki K, Matsumoto T, Aono H, et al. Relationship between epicardial fat measured by 64multidetector computed tomography and coronary artery disease. Clin Cardiol. 2011; 34: 166-171.

13. Yamashita K, Yamamoto MH, Ebara S, et al. Association between increased epicardial adipose tissue volume and coronary plaque composition. Heart Vessels. 2014; 29: 569-577. 
14. Ueno K, Anzai T, Jinzaki M, et al. Increased epicardial fat volume quantified by 64-multidetector computed tomography is associated with coronary atherosclerosis and totally occlusive lesions. Circ J. 2009; 73: 1927-1933.

15. Yu W, Liu B, Zhang F, et al. Association of Epicardial Fat Volume With Increased Risk of Obstructive Coronary Artery Disease in Chinese Patients With Suspected Coronary Artery Disease. JAHA. 2021; 10: $\mathrm{e} 018080$.

16. Gorter PM, de Vos AM, van der Graaf Y, et al. Relation of epicardial and pericardial fat to coronary atherosclerosis and coronary artery calcium in patients undergoing coronary angiography. Am J Cardiol. 2008; 102: 380-385.

17. Saura DO, Oliva MJ, Rodriguez D, et al. Reproducibility of echocardiographic measurements of epicardial fat thickness. Int J Cardiol. 2010; 141: 311-312.

18. Sinha SK, Thakur R, Jha MJ, et al. Epicardial Adipose Tissue Thickness and Its Association With the Presence and Severity of Coronary Artery Disease in Clinical Setting: A Cross-Sectional Observational Study. Journal of Clinical Medicine Research. 2016; 8(5): 410-419.

19. Shambhu SK, Desai N, Sundaresh N, et al. Study of correlation between epicardial fat thickness and severity of coronary artery disease. 2020; 72(5): 445-447.

20. Verma B, Katyal D, Patel A, et al. Relation of systolic and diastolic epicardial adipose tissue thickness with presence and severity of coronary artery disease (The EAT CAD study). J. Family Med Prim Care. 2019; 8: 1470-1475.

21. Erkan AF, Tanindi A, Kocaman SA, et al. Epicardial adipose tissue thickness is independently related to the coronary atherosclerotic burden determined by the SYNTAX and Gensini score. Eur Heart J. 2013; 34: 3084 .

22. Iacobellis G, Willens HJ, Barbaro G, et al. Threshold values of high risk echocardiographic epicardial fat thickness. Obesity 2008; 16: 887-892.

23. Wang TD, Lee WJ, Shih FY, et al. Relations of epicardial adipose tissue measured by multidetector computed tomography to components of the metabolic syndrome are region-specific and independent of anthropometric indexes and intraabdominal visceral fat. J Clin Endocrinol Metab. 2009; 94: 662-669.

24. Moharram MA, Aitken-Buck HM, Reijers R, et al. Correlation between epicardial adipose tissue and body mass index in New Zealand ethnic populations. NZ Med J. 2020; 133: 22-32.

Table 1: Baseline characteristics of study participants

\begin{tabular}{lll}
\hline Characteristics & CAD $(\mathbf{n}=\mathbf{3 8})$ & No CAD $(\mathbf{n = 5 9})$ \\
\hline Age (mean \pm SD) & $59.18 \pm 8.56 \mathrm{mg} / \mathrm{dL}$ & $54.15 \pm 8.23 \mathrm{mg} / \mathrm{dL}$ \\
Males (n, \%) & $21(55.3 \%)$ & $29(49.2 \%)$ \\
Females (n, \%) & $17(44.7 \%)$ & $30(50.8 \%)$ \\
HTN (n, \%) & $28(73.7 \%)$ & $46(78 \%)$ \\
DM (n, \%) & $13(34.2 \%)$ & $19(32.2 \%)$ \\
Dyslipidaemia (n, \%) & $14(36.8 \%)$ & $18(30.5 \%)$ \\
Tobacco use (n, \%) & $7(18.4 \%)$ & $5(8.5 \%)$ \\
Obesity (n, \%) & $7(18.4 \%)$ & $14(23.7 \%)$ \\
CAC (0) (n, \%) & $13(34.2 \%)$ & $48(81.4 \%)$ \\
CAC (0-99.99) (n, \%) & $14(36.8 \%)$ & $10(17 \%)$ \\
CAC ([?]100) (n, \%) & $11(29 \%)$ & $1(1.7 \%)$ \\
TC (mean \pm sd) & $165.50 \pm 52.76 \mathrm{mg} / \mathrm{dl}$ & $167.62 \pm 49.20 \mathrm{mg} / \mathrm{dl}$ \\
LDL (mean \pm sd) & $99.43 \pm 32.89 \mathrm{mg} / \mathrm{dl}$ & $109.37 \pm 39.60 \mathrm{mg} / \mathrm{dl}$ \\
TG (mean \pm sd) & $180.11 \pm 162.74 \mathrm{mg} / \mathrm{dl}$ & $165.92 \pm 101.86 \mathrm{mg} / \mathrm{d}$ \\
BMI (mean \pm sd) & $25.82 \pm 5.02$ & $26.18 \pm 5.68$ \\
QRISK3 score (risk of heart attack/stroke in 10 years) (median \pm range) & $17.80 \pm 11.90 \%$ & $12.38 \pm 8.56 \%$ \\
\hline
\end{tabular}

* Statistically significant ( $\mathrm{p}$ value $<0.05$ ) 
$[\mathrm{HTN}=$ hypertension, $\mathrm{DM}=$ diabetes mellitus, $\mathrm{CAD}$ : coronary artery disease; $\mathrm{CAC}=$ coronary artery calcium score, $\mathrm{TC}=$ total cholesterol, $\mathrm{LDL}=$ low density lipoprotein, $\mathrm{TG}=$ triglyceride, $\mathrm{BMI}=$ body mass index]

Table 2: Correlation of epicardial adipose tissue thickness and epicardial adipose tissue volume with coronary artery disease:

\begin{tabular}{llll}
\hline Variable & CAD $(\mathbf{n}=\mathbf{3 8})$ & NO CAD $(\mathbf{n}=\mathbf{5 9})$ & P value \\
\hline EAT-T (PLAX) & $4.82 \pm 1.31 \mathrm{~mm}$ & $4.06 \pm 1.25 \mathrm{~mm}$ & $0.005^{*}$ \\
Indexed EAT-T (PLAX) & $2.91 \pm 0.96 \mathrm{~mm} / \mathrm{m}^{2}$ & $2.35 \pm 0.74 \mathrm{~mm} / \mathrm{m}^{2}$ & $0.002^{*}$ \\
EAT-T (PSAX) & $4.79 \pm 1.32 \mathrm{~mm}$ & $4.09 \pm 1.24 \mathrm{~mm}$ & $0.01^{*}$ \\
Indexed EAT-T (PSAX) & $2.89 \pm 0.97 \mathrm{~mm} / \mathrm{m}^{2}$ & $2.37 \pm 0.74 \mathrm{~mm} / \mathrm{m}^{2}$ & $0.04^{*}$ \\
EAT-V (CTA) & $129.55 \pm 31.51 \mathrm{~cm}^{3}$ & $104.42 \pm 30.19 \mathrm{~cm}^{3}$ & $<0.001^{*}$ \\
Indexed EAT-V (CTA) & $77.05 \pm 19.49 \mathrm{~cm}^{3} / \mathrm{m}^{2}$ & $60.01 \pm 16.50 \mathrm{~cm}^{3} / \mathrm{m}^{2}$ & $<0.001^{*}$ \\
\hline
\end{tabular}

* Statistically significant ( $\mathrm{p}$ value $<0.05)$

[EAT-T: epicardial adipose tissue thickness; EAT-V: epicardial adipose tissue volume; PLAX: parasternal long-axis view; PSAX: parasternal short-axis view; CTA: computed tomography angiography; CAD: coronary artery disease]

Table 3: Correlation of epicardial adipose tissue volume with epicardial adipose tissue thickness:

\begin{tabular}{lllll}
\hline Variable & EAT-T (PLAX) & EAT-T (PLAX) & EAT-T (PSAX) & EAT-T (PSAX) \\
\hline & $\mathbf{r}$ & p value & r & p value \\
EAT-V (CTA) & 0.69 & $<0.001^{*}$ & 0.67 & $<0.001^{*}$ \\
\hline
\end{tabular}

* Statistically significant $(\mathrm{p}$ value $<0.05$ )

[r: Pearson correlation coefficient; EAT-T: epicardial adipose tissue thickness; EAT-V: epicardial adipose tissue volume; PLAX: parasternal long-axis view; PSAX: parasternal short-axis view; CTA: computed tomography angiography]

Table 4: Correlation of indexed epicardial adipose tissue volume with indexed epicardial adipose tissue thickness:

\begin{tabular}{lllll}
\hline Variable & Indexed EAT-T (PLAX) & Indexed EAT-T (PLAX) & Indexed EAT-T (PSAX) & I \\
\hline & $\mathrm{r}$ & $\mathrm{p}$ value & $\mathrm{r}$ & $\mathrm{p}$ \\
Indexed EAT-V (CTA) & 0.75 & $<0.001^{*}$ & 0.73 & $<$ \\
\hline
\end{tabular}

* Statistically significant ( $\mathrm{p}$ value $<0.05$ )

[r: Pearson correlation coefficient; EAT-T: epicardial adipose tissue thickness; EAT-V: epicardial adipose tissue volume; PLAX: parasternal long-axis view; PSAX: parasternal short-axis view; CTA: computed tomography angiography]

Table 5: Univariate regression analysis for the effect of epicardial adipose tissue thickness on coronary artery disease:

\begin{tabular}{llll}
\hline Variable & OR & $\mathbf{9 5 \%}$ CI & P value \\
\hline EAT-T (PLAX) & 2.50 & $1.08-5.79$ & $0.03^{*}$
\end{tabular}




\begin{tabular}{llll}
\hline Variable & OR & 95\% CI & P value \\
\hline Indexed EAT -T (PLAX) & 2.88 & $1.24-6.71$ & $0.01^{*}$ \\
EAT -T (PSAX) & 2.24 & $0.97-5.14$ & $0.05^{*}$ \\
Indexed EAT -T (PSAX) & 2.68 & $1.16-6.22$ & $0.02^{*}$ \\
\hline
\end{tabular}

* Statistically significant $(\mathrm{p}$ value $<0.05$ )

[EAT-T: epicardial adipose tissue thickness; PLAX: parasternal long-axis view; PSAX: parasternal short-axis view]

Table 6: Multivariate regression analysis for effect of epicardial adipose tissue thickness (absolute and indexed) on coronary artery disease:

\begin{tabular}{|c|c|}
\hline VARIABLE & OR $95 \%$ CI P value \\
\hline QRISK 3 EAT-T (PLAX) & $1.11 .00-1.100 .03 * 2.81 .15-6.570 .02 *$ \\
\hline QRISK 3 Indexed EAT-T (PLAX) & $1.11 .00-1.100 .04 * 5.42 .17-13.55<0.001 *$ \\
\hline QRISK 3 EAT-T (PSAX) & $1.11 .00-1.100 .03^{*} 2.61 .07-6.030 .03^{*}$ \\
\hline QRISK 3 Indexed EAT -T (PSAX) & $1.11 .01-1.100 .03 * 3.31 .37-7.930 .01 *$ \\
\hline
\end{tabular}

* Statistically significant $(\mathrm{p}$ value $<0.05)$

[EAT-T: epicardial adipose tissue thickness; PLAX: parasternal long-axis view; PSAX: parasternal short-axis view]

Figure Legends:

Figure 1: Quantification of epicardial adipose tissue thickness (arrow) by transthoracic echocardiography, parasternal long axis view. [Ao: aorta; RV: right ventricle; LV: left ventricle; LA: left atrium]

Figure 2: Quantification of epicardial adipose tissue thickness (arrow) by transthoracic echocardiography, parasternal short axis view. [RV: right ventricle; LV: left ventricle]

Figure 3: Receiver operating characteristic (ROC) area under curve (AUC) for epicardial adipose tissue. [EAT-T: epicardial adipose tissue thickness; EAT-V: epicardial adipose tissue volume; PLAX: parasternal long-axis view; PSAX: parasternal short-axis view; MSCT: multi-slice computed tomography angiography] 

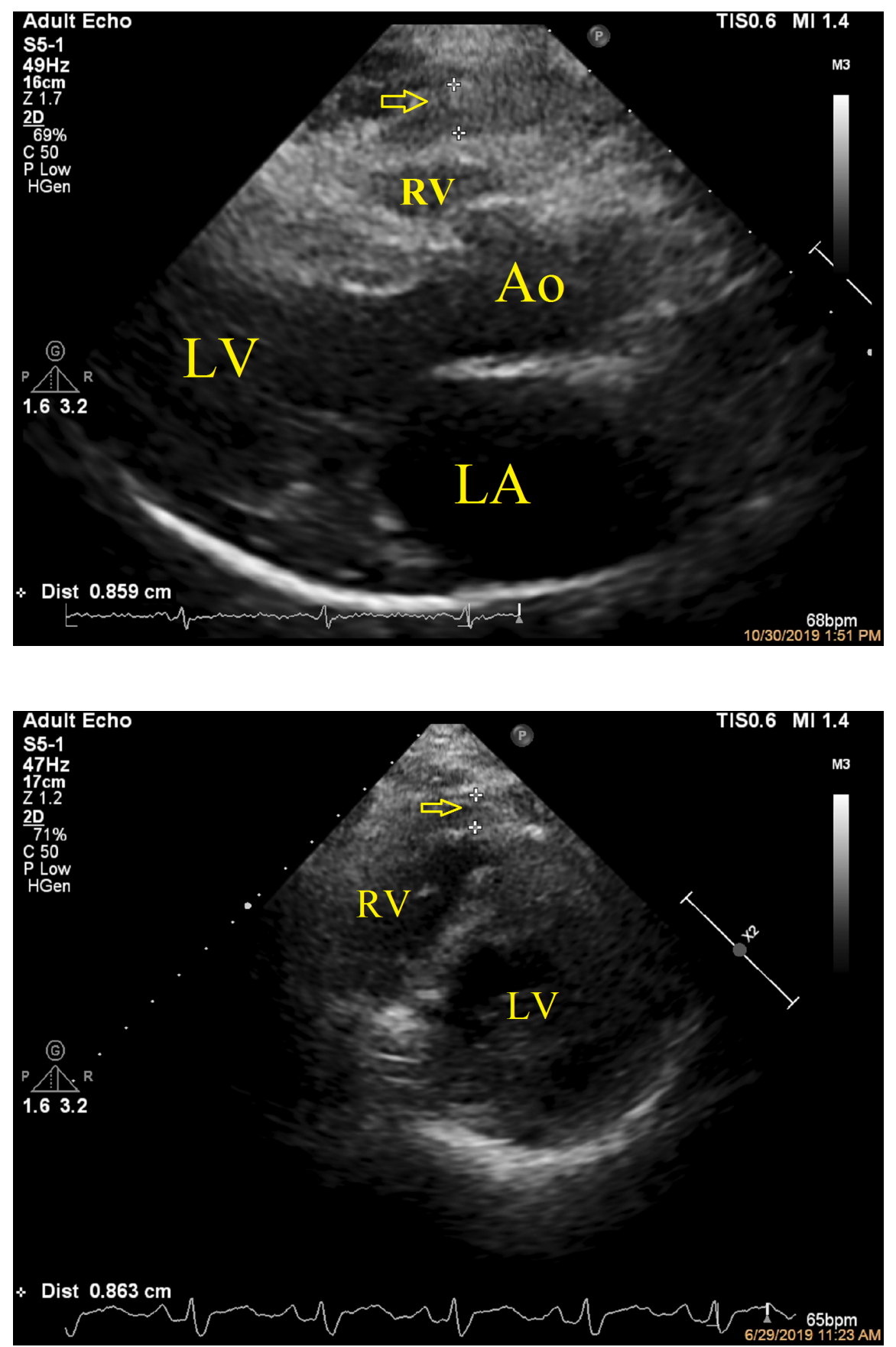


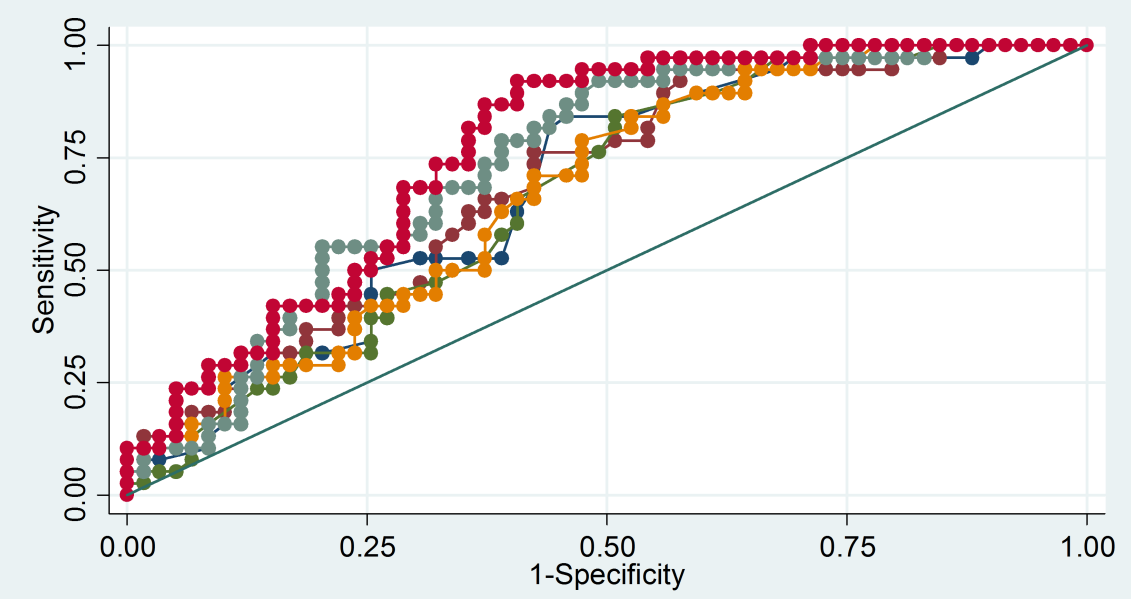

$\begin{array}{ll}\longrightarrow & \text { EAT-T(PLAX) ROC area: } 0.68 \\ & \text { EAT-T(PSAX) ROC area: } 0.66 \\ & \text { EAT-V(MSCT) ROC area: } 0.73 \\ \text { Reference } & \text { EAT-T(PSAX) INDEXED ROC area: } 0.67 \\ & \end{array}$

